\title{
Development of an On-Line System for Precision Dimensional Measurements in Electrical Steels using Approach to Ferromagnetic Saturation
}

\author{
Gholamhossein Shirkoohi, Member, IEEE \\ School of Engineering \\ London South Bank University \\ London SE1 0AA, UK \\ Email: maziar.shirkoohi@1sbu.ac.uk
}

\begin{abstract}
A system based on a measurement technique magnetisation near saturation is presented that allows high precision measurement of dimensional parameters of iron alloys. Extensive measurements in electrical steel laminations show high accuracy in measurement of cross section area of the strips. The technique uses approach to saturation magnetisation which has previously been shown to be dependent on alloying content of the materials. This system is proposed for on-line measurement of cross sectional area and thickness of electrical steels, and other types of ferromagnetic strip metals.
\end{abstract}

Keywords-approach to saturation; electrical steel; iron alloy; alloying content; cross section area

\section{INTRODUCTION}

The high permeability and low iron loss characteristics of silicon iron alloys makes them ideal for construction of laminated cores of transformers, generators and motors. During their manufacture, it is highly desirable to assess their magnetic properties whilst emerging from the production lines. This very much depends on the accuracy with which their cross section area is measured. One of the most critical performance indicators for laminated electrical steels strips is their iron loss, which needs to be determined at maintained specific $B_{P E A K}$ values. This can only be measured with high precision, if an accurate cross section area is known [1]. This is also desirable for a host of long products such as sheet metals, metal beams, rods, etc.

The measurement technique described here provides the means for accurate dimensional measurement of ferromagnetic materials using their saturation, which is closely tied to their alloying contents. This also ties in with the recent increase in the interests in measurements in the saturation range [2-5]. Although magnetic saturation has not been the subject of interest in the past, modern techniques in control and operation of rotating machines has lead to the development of new methods for characterizing these materials [6].

Other research work report heavy saturation in transformer cores, used in renewable energy systems [7], and in welding transformers, generated by dc-dc converters $[8,9]$. Apart from this other related work includes utilizing magnetisation curves in order to predict losses in three-phase Induction Motors [10].

Saturation effects have also been subject of studies in geometry instigated unbalanced phase currents [11] and nondestructive measurement of surface-hardening depth of induction-hardened and carburized steel rods [12]. These are just a few examples of saturation effects in modern electrical equipment. There have also been recent studies carried out in following the law of approach to saturation [13] in the region near the peak values of the external magnetic field, where the specimen approaches magnetic saturation. This approach is used as the basis for the work presented in this paper.

Traditional techniques for thickness measurement on moving strip in production lines would include contact micrometers, ultrasonic devices, nucleonic radiation absorption gauges etc. X-ray and $\gamma$-ray techniques also became popular, but X-rays involve high voltage systems and $\gamma$-rays need isotope sources. Neither of these is cheap or convenient and the width of the portion of strip actually interrogated is often too small compared to its full width, so that across-strip variation of thickness is ignored [14]. Various magnetic techniques have been considered for thickness assessment, mainly relating to magnetisation through the strip thickness. Such a method suffers badly from flux spreading and the very high demagnetising factors involved.

\section{SYSTEMATIC COMPARISONS}

Theoretically the cross sectional area of a strip can be assessed by measuring the amount of magnetic polarisation $\left(\mathrm{J}_{\text {sat }}\right)$ generated by fully saturating it. But there are problems associated with these. If the material is not pure iron, e.g. it has significant amounts of alloying elements, the precise effect of alloying elements on $\mathrm{J}_{\text {sat }}$ should be determined. Also if deep saturation is achieved, the effects of crystal structure, grain size and morphology on $\mathrm{J}_{\text {sat }}$ should be mitigated satisfactorily. Effect of temperature should also be considered.

In the case of Epstein grading and single sheet off-line testing cross sectional area is derived from mass size and 
density. Density also varies with alloy level and should be adjusted accordingly. Conventional formulae are used for this in industry. Uncertainties arise from the use of conventional compositions and conventional densities. These are accepted in the industry and are generally no greater than $\sigma=0.25 \%$. When radiation gauges are used for on line thickness (and, via width, cross sectional area) measurement, a careful assessment has to be made of the practicability. Conventional densities are replaced with conventional radiation opacities. These would relate the systematic variation of opacity with alloy content to the statistical impact of using grade-specific conventional opacities. This has been well carried out within the steel industry for $\gamma$-ray thickness measurement. The only serious defect lies in the limitation of the radiation gauges to be confined to a region no larger than $2-3 \mathrm{~cm}$ in diameter.

\section{SATURATION METHOD - THEORETICAL APPROACH}

It can be shown that a single crystal of iron can be saturated in any direction by the application of some $50 \mathrm{kA} / \mathrm{m}$ of effective applied field. This is set by energy considerations, since for a crystal whose easy direction is not in the direction of magnetisation applying progressively larger fields adds to two forms of energy storage; that due to magnetocrystalline anisotropy, and that due to the interaction between the applied field and the field internal to the crystal. In the real polycrystalline material the approach to $\mathrm{J}_{\text {sat }}$ is impeded by crystal defects, grain size and shape and a variety of other factors.

Empirical laws describing the approach to saturation can be applied. For this case Lamont's law [15] states:

$\chi=C\left(J_{s a t}-J\right)$ where $\chi$ is the material susceptibility and $C$ is a constant

Hence the susceptibility falls rapidly as $\mathrm{J}_{\text {sat }}$ is approached. Actual impact of alloying elements on $\mathrm{J}_{\text {sat }}$ was investigated here. Overall, the addition of silicon and aluminum to iron appears to have a simple diluting effect.

\section{EXPERIMENTAL SETUP AND MEASUREMENTS}

A good deal of detail on the magnetising system used for measurements on Epstein size samples was previously reported [16]. Fig. 1 shows the block diagram for the overall system. By saturation magnetic polarisation method, crosssectional areas of the electrical steels with known saturation magnetic polarisation are assessed using the flux reversals produced when the samples are strongly saturated. Accuracy of the method depends directly on the determination of saturation magnetic polarisation. In the study, the dependences of saturation magnetic polarisation of electrical steels on various effects, such as temperature, the depth of magnetic saturation and alloying contents, are firstly determined experimentally and then analysed theoretically. The experimental study was made by measuring the magnetic properties of the Epstein strips using an air-cored magnetic tester as shown in Fig. 1 (inset). The applied magnetic field is produced by a coil energised using a sinusoidal voltage of 50 $\mathrm{Hz}$ from the mains. The exciting current is switch on/off using a computer-controlled thyristor assembly. Intensity of the applied magnetic field is determined from the exciting current using a Hall-effect current transducer. Frequency of the exciting current is measured by counting a $1 \mathrm{MHz}$ clock in one cycle of the current. Zero-crossing of the exciting current, which is detected using a comparator, is used to start, stop and control the whole process of measurement. Magnetic

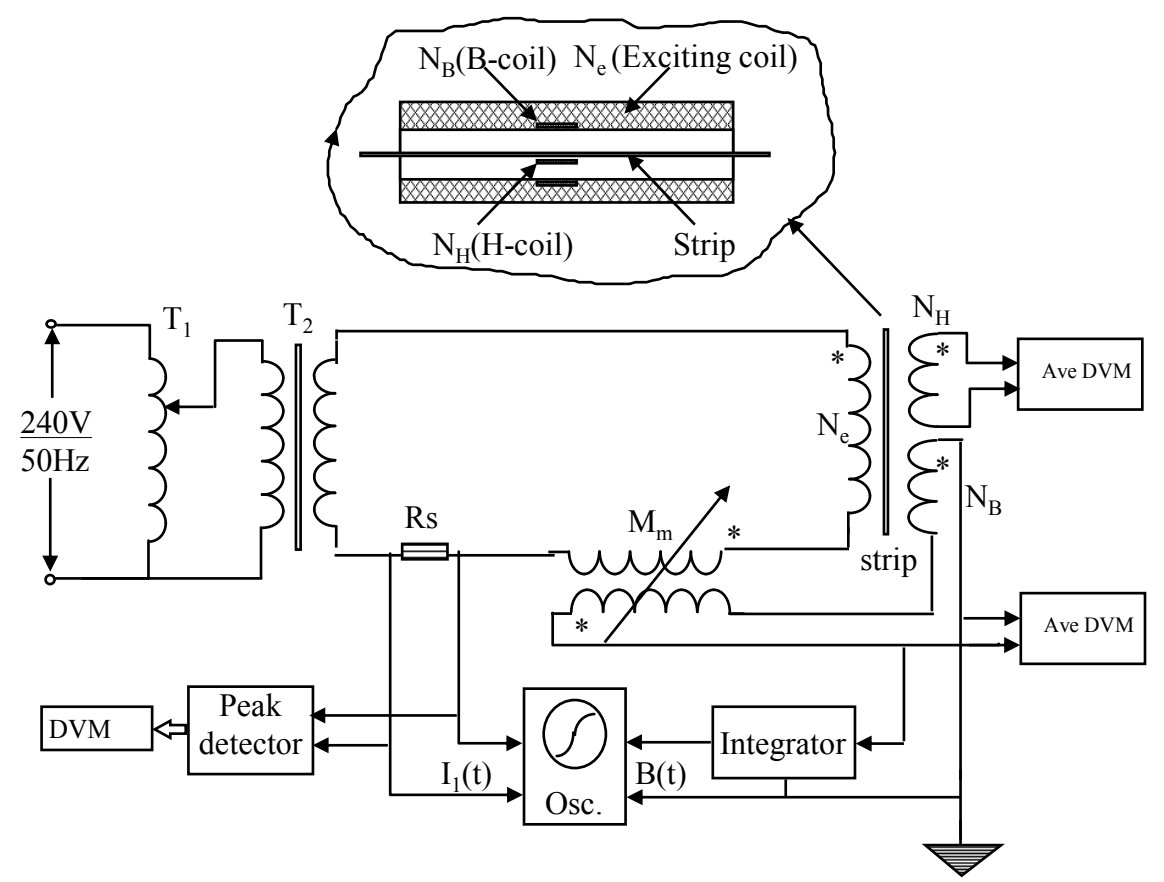

Fig 1 The set-up for measuring magnetic properties of electrical strips 
polarisation of the strips is determined by sampling and integrating the differential induced voltage from the B-coil, $N_{B}$, wound inside the exciting coil, and a compensating mutual inductor, $\boldsymbol{M}_{\boldsymbol{B}}$, located outside. Former of the B-coil is made from low thermal expansion material so that the effect of temperature variation on it is negligible. The demagnetising field is measured using an H-coil placed next to the surface of the strip under testing and the induced voltage due to the applied magnetic field is compensated using another mutual inductor, $\boldsymbol{M}_{\boldsymbol{H}}$.

In the study, to saturate the part of an Epstein specimen inside the exciting coil, a minimum applied magnetic field of $50 \mathrm{kA} / \mathrm{m}$ is required everywhere in the plate [16]. This requirement is met with by winding $N_{e}=3060$ turns of coil on a $200 \mathrm{~mm}$ long former. Average values of the width and the height of the coil are $54 \mathrm{~mm}$ and $31 \mathrm{~mm}$ respectively. If all the other parameters of the exciting coil remain the same, but its width is increased from $54 \mathrm{~mm}$ to $1 \mathrm{~m}$ and height from $31 \mathrm{~mm}$ to $50 \mathrm{~mm}$ to measure the $1 \mathrm{~m}$ wide electrical steel sheets. Strength of the applied magnetic field at both ends of the coil is approximately half of the strength at the middle. Maximum strength of the applied magnetic field near both sides of the coil is due to the increasing contribution of magnetic field by the current-carrying wires on both sides. When width of a rectangular coil is much bigger than its height, magnetic field in the central area of the coil is mainly due to the currentcarrying wires on the upper and lower plates of the coil, and therefore does not decrease obviously with the increase of the coil width.

\section{A. Measurement of saturation magnetic polarisation}

Relationship between the induced voltage from the B-coil and magnetic flux density of the sample is determined using

$e_{B}(t)=N_{B} \frac{d \Phi}{d t}=N_{B}\left[A_{s} \frac{d B(t)}{d t}+\mu_{0}\left(A_{B}-A_{s}\right) \frac{d H_{t}(t)}{d t}\right]$

Where $\boldsymbol{A}_{\boldsymbol{B}}$ and $\boldsymbol{N}_{\boldsymbol{B}}$ are the cross-sectional area and the number of turns of the B-coil. $A_{S}$ is the cross sectional area of the sample. $B(t), \mathrm{y}$-component of the instantaneous magnetic flux density of the specimen, is determined using

$$
B(t)=M_{s}(t)+\mu_{0} H(t)
$$

Total magnetic field $\boldsymbol{H}_{\boldsymbol{t}}(\boldsymbol{t})$ is the difference between the applied magnetic field $\boldsymbol{H}_{\boldsymbol{a}}(\boldsymbol{t})$ and the demagnetising field, $H_{d}(t)$ :

$$
H_{t}(t)=H_{a}(t)-H_{d}(t)
$$

Substituting equations (3) and (4) into (2), then

$$
e_{B}(t)=N_{B}\left[A_{S} \cdot \frac{d J(t)}{d t}+\mu_{0} A_{B} \cdot \frac{d H_{a}(t)}{d t}-\mu_{0} A_{B} \cdot \frac{d H_{d}(t)}{d t}\right]
$$

Equation (5) shows that the induced voltage from the Bcoil consists of three parts: the first part is related to the magnetic polarisation and cross-sectional area of the strip to be tested. The second part is due to the applied magnetic field and can be compensated using a mutual inductor whose primary winding is connected in serial addition with the exciting coil and the secondary winding is connected in serial opposition with the B-coil. The third part is due to the demagnetising field produced by the non-uniform magnetisation in the sample volume and the discontinuance of magnetic polarisation on the boundary. It is compensated by measuring the demagnetising field using an H-coil placed next to the sample.

When a complete compensation has been made, the net induced voltage from the B-coil is only related to the magnetic polarisation and cross-sectional area of the sample, i.e.,

$e_{B}(t)=N_{B} A_{S} \frac{d J(t)}{d t}$

Saturation magnetic polarisation of the sample is essentially determined by sampling the instantaneous values of the induced voltage and then integrating them using a software integrator. Calibration of the measurement is made by measuring its full-wave rectified mean values using a $0.1 \%$ mean responding multimeter.

Mean value of the full-wave rectified induced voltage $\boldsymbol{e}_{B}(\boldsymbol{t})$ is:

$$
\begin{aligned}
\overline{E_{B}} & =\frac{1}{T_{e}} \int_{0}^{T_{e}} e_{B}(t) \cdot d t=\frac{2}{T_{e}} \int_{t_{2}}^{t_{3}}\left[N_{B} \cdot A_{s} \cdot \frac{d J(t)}{d t}\right] \cdot d t \\
& =\frac{2}{T_{e}} N_{B} A_{s} \int_{-J_{S}}^{J_{S}} d J(t)=4 f_{e} N_{B} A_{s} J_{s}
\end{aligned}
$$

$\overline{\boldsymbol{E}_{\boldsymbol{B}}}$ is hence only proportional to cross sectional area or saturation magnetic polarisation of the specimen.

Since the reading on the ac function of a mean responding multimeter is $\boldsymbol{r m s}$ calibrated for sinusoidal signal ratio of the reading to the average value of the signal to be measured is a constant of $\eta=\pi / 2 \sqrt{2}$. So the relationship between value $\overline{\boldsymbol{E}_{\boldsymbol{B}}}$ and the reading of a mean responding multimeter is:

$$
\overline{E_{B}}=\frac{V_{r}}{\eta}=\frac{2 \sqrt{2}}{\pi} V_{r}
$$

Substituting equation (8) into equation (7), saturation magnetic polarisation can be described as a function of $\boldsymbol{V}_{\boldsymbol{r}}$ :

$$
J_{S}=\frac{\sqrt{2} V_{r}}{2 \pi f_{e} A_{S} N_{B}}
$$

Frequency $f_{e}$ of the exciting current can be precisely measured. Cross-sectional area $\boldsymbol{A}_{\boldsymbol{S}}$ of the sample is obtained using the density method as described in chapter two.

\section{B. Measurement of the demagnetising field}

Owing to the continuity of the tangential component of magnetic field at the boundary of two media, the 
demagnetising field inside the sample is measured using an $\mathrm{H}$ coil placed just outside it.

The induced voltage from the $\mathrm{H}$-coil is determined by

$e_{H}(t)=\mu_{0} N_{H} A_{H} \frac{d H_{t}(t)}{d t}$

and

$$
H_{t}(t)=H_{a}(t)-H_{d}(t)
$$

hence,

$$
e_{H}(t)=\mu_{0} N_{H} A_{H}\left(\frac{d H_{a}(t)}{d t}-\frac{d H_{d}(t)}{d t}\right)
$$

Where $\boldsymbol{A}_{\boldsymbol{H}}$ and $\boldsymbol{N}_{\boldsymbol{H}}$ are the cross sectional area and the number of turns of the H-coil.

The part due to the applied magnetic field is compensated using a mutual inductor $\left(\boldsymbol{M}_{\boldsymbol{H}}\right)$ whose primary winding is connected in serial addition with the exciting coil and the secondary winding is connected in serial opposition with the H-coil.

The net induced voltage that is just corresponding to the demagnetising magnetic field is then:

$$
e_{H}(t)=-\mu_{0} N_{H} \cdot A_{s} \cdot \frac{d H_{d}(t)}{d t}
$$

Temporary values of the demagnetising field are obtained by integrating the net induced voltage using a software integrator. Then,

$$
E_{H}(t)=-\mu_{0} N_{H} A_{s} H_{d}(t)
$$

Instantaneous values of the demagnetising field, related to the respective values of the applied magnetic field, are obtained by sampling them simultaneously.

\section{Measurement of exciting current}

The measurement of exciting current is carried out using a Hall-effect current transducer. When a current carrying wire passes through a magnetic core, current $\boldsymbol{I}_{\boldsymbol{e}}$ in the wire will produce a proportional magnetic field at the gap of the core. Magnetic field in the gap is detected using a Hall-effect element. Hall voltage across this element is linearly proportional to the magnetic field and hence the current $\boldsymbol{I}_{\boldsymbol{e}}$ flowing in the wire. Sensitivity of the transducer can be adjusted by changing the number of times that the current

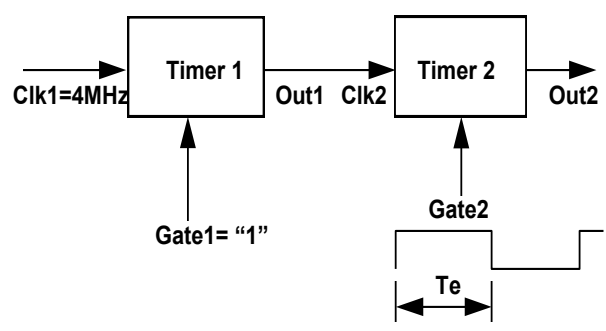

Fig. 2 The measurement of frequency by cascading two timers carrying wire passes through the centre of the core.

Main advantages of the transducer are its electrical isolation between the current carrying wire and the Hall-effect element, as well as its wide band of frequency that is required in the measurement of exciting current with some level of distortion.

A Hall-effect current transducer was used in the experimental set-up. This current transducer has the following specification; a range of $0-200 \mathrm{~A}$, accuracy of $1 \%$ and the frequency of operation between dc to $25 \mathrm{kHz}$.

\section{Measurement of frequency}

Frequency of the exciting current is measured indirectly by counting a pulse single of fixed frequency in one cycle of the exciting current using the circuit shown in Fig. 2. Both counter 1 and counter 2 are programmed into mode 3 . The output of counter 1 , which is generated by $1 / 4$ dividing a stabilised 4 $\mathrm{MHz}$ clock, is used as a clock for counter 2. The time constant of counter 2 is set as \$FFFF, and its gate is switched by a square wave "Gate 2", obtained by $1 / 2$ dividing the frequency of exciting current. Owing to the high level engagement in the first half cycle of "Gate 2", counter 2 starts counting. In the second half cycle, when the low-level of the $1 / 2$ square wave is detected by the host computer, counter 2 is stopped and reading of the counted number is carried out.

Relationship between the counted number $N_{C}$ and frequency $f_{e}$ of the exciting current is:

$$
N_{c} \cdot T_{c}=T_{e}=1 / f_{e} \quad \text { or } \quad f_{e}=\frac{1}{N_{c} T_{c}}
$$

where: $T_{c}$ is the cycle for "Clk 2 ".

In the experimental set-up, frequency of the exciting current is $50 \mathrm{~Hz}$, while the frequency of "Clk 2" is $1 \mathrm{MHz}$. So, in one cycle of the exciting current, the counted number is about $N_{C}=2 \times 10^{4}$, and hence resolution of the frequency measurement is $\pm\left(1 / 2 \times 10^{4}\right)$ or $\pm \mathbf{0 . 0 0 5}$ per cent.

\section{E. A/D converter}

Fig. 3 shows the A/D converter and related electronics used in the experimental set-up. A 12-bit A/D converter is

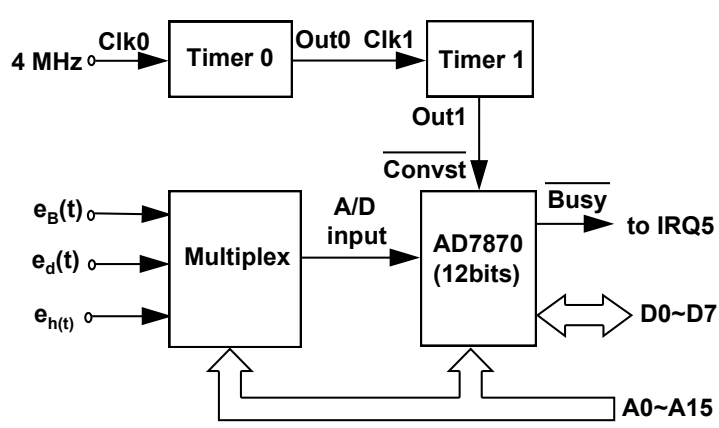

Fig. 3 The A/D converter and the associated control circuitry 


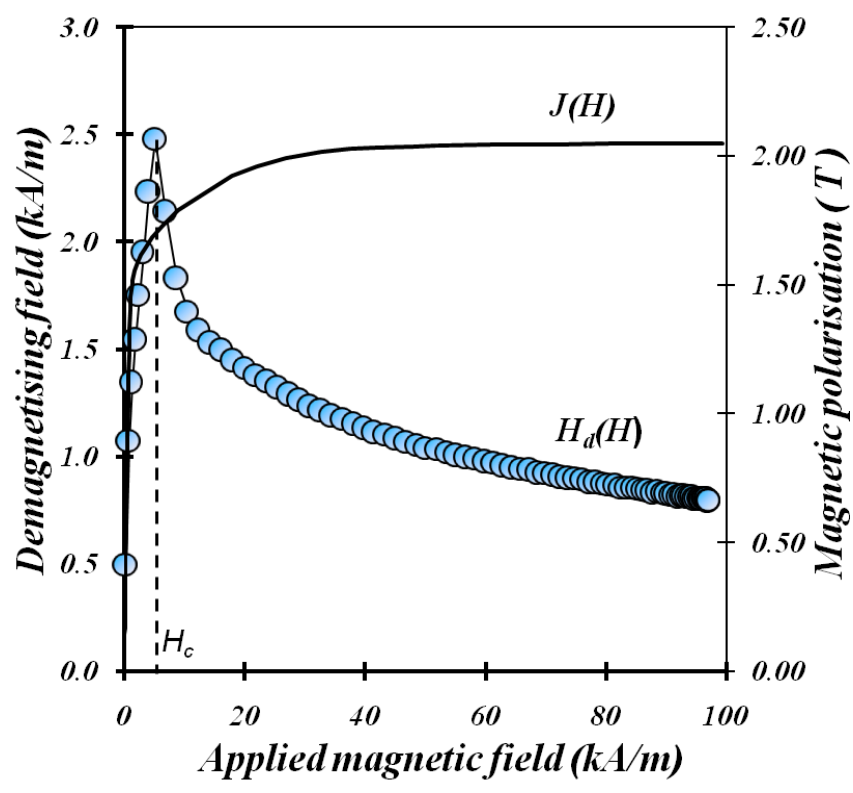

Fig. 4. Variation of demagnetising field with magnetic field intensity, measured in the centre of a $150 \mathrm{~mm}$ long, $0.65 \mathrm{~mm}$ thick, $0.1 \%$ silicon non-oriented steel sample. $J(H)$ is magnetic polarisation and $H d(H)$ is the demagnetising Field, both dependant on applied magnetic field $H$.

wired for Mode 1 operation with parallel output format in bytes. Its conversion is initiated by a low going pulse on $\overline{\text { Convst }}$, generated by programming and cascading counter 0 and counter 1 of a 8253 interval timer on the same board in Mode 3 and Mode 2 separately to give a sampling rate of 40 $\mathrm{kHz}$. At the end of a conversion, $\overline{\text { Busy }}$ goes low, and generates an interrupt request IRQ5 to the host computer. When it is acquainted, the host computer executes an interrupt service subroutine and read out the data converted in two steps, the reading of higher 4 bits being followed by the lower 8 bits. Since the 12 bit data is coded in the form of 2 s complement with the most significant bit inverted, a transfer program has been used to transfer this into pure binary.

Three input voltages, $\boldsymbol{e}_{B}(t), \boldsymbol{e}_{h}(t)$ and $\boldsymbol{e}_{d}(t)$ of the A/D converter are sampled alternatively by switching a multiplex. Sampling rate for each channel is therefore one-third of the A/D sampling rate, i.e., $13 \mathrm{kHz}$, and sampling points for each channel is approximately 260 for the input frequency of 50 $\mathrm{Hz}$. The experimental system was devised so that appropriate measurements could be made on Epstein-strip sized samples and data derived which would clarify the scope of measurement needed and enable a data base to be assembled so that magnetic saturation methods could be assessed in comparison with weighing and $\gamma$-ray absorption techniques. This was previously described in detail [17]. The test frame comprises a magnetising coil, B coil and $\mathrm{H}$ coil. Flux closure yokes were not necessary and were not used. The magnetising coil is able to deliver some $100 \mathrm{kA} / \mathrm{m}$ at its centre. The B coil was connected to an air flux compensating mutual inductor so that flux from the sample was detected but not air flux from the applied field, i.e. J only. An H coil placed near the sample surface was also connected to a mutual inductor so that when the flux to this $\mathrm{H}$ coil was balanced out (sample absent), the demagnetising field (sample present) would be detected by it, free of $\mathrm{H}$ applied.

This arrangement enabled close monitoring of the size of the demagnetising field arising, as the sample geometry is changed. This greatly facilitated the confidence in the design of the measurement system. Demagnetising factors can be either calculated or measured as may be convenient in each case. When using a fully compensated system the flux recorded is from sample polarisation alone and excludes the magnetising air flux and the effects of the demagnetising field.

Excitation was at $50 \mathrm{~Hz}$, the supply waveform being uncritical, and the quantities measured:

$\boldsymbol{J}_{\text {sat }}$ was calculated from basic formula (Faraday's Law):

$V=4 J_{\text {sat }} n f A$

where $\quad \boldsymbol{V}=$ average voltage of compensated B coil $\boldsymbol{n}=$ number of turns of B coil

$\boldsymbol{f}=$ supply frequency

$\boldsymbol{A}=\mathrm{B}$ coil cross section

\section{F. Preparation of samples.}

A large number of samples were assembled from a variety of Electrical Steel grades and checked for thickness at various points by $\gamma$-ray absorption. Their composition was determined by chemical analysis. Densities based on the known impact of the alloying elements involved were assigned to each sample. Samples were carefully treated so that guaranteed exactness of width, sample to sample, could be assumed.

\section{G. Demagnetising field.}

Measurements of the demagnetising fields were made for all the samples. This is shown for a typical sample of Epstein width $(30 \mathrm{~mm})$ in Fig. 4. The field is asymptotic to some 0.8

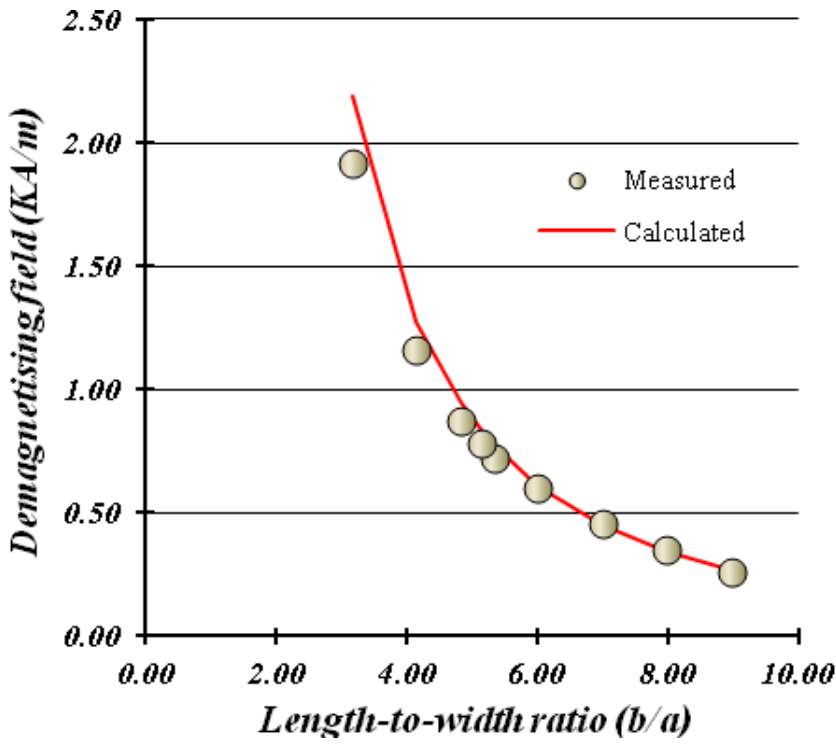

Fig. 5.Variation of demagnetising field with sample aspect ratio (length to width) for $0.1 \%$ silicon non-oriented steel. 
$\mathrm{kA} / \mathrm{m}$ at $\mathrm{H}$ applied $100 \mathrm{kA} / \mathrm{m}$. Fig. 5 shows how the demagnetising field varies with length to width ratio, clearly showing that the demagnetising field, due to shape anisotropy, becoming very large when the length of the specimen become smaller in comparison to the of the width, as would be expected, giving rise to elevation of uncertainties between the calculated and the measurement data. Calculation and measurement are otherwise seen to be in good agreement.

From these results it is clear that for an industrial device the demagnetising factor can either be; ignored for a long sample (this would apply on production line), or calculated for a particular device and shape of the test specimen, or continuously compensated using a compensating $\mathrm{H}$ coil and mutual inductor.

\section{H. Approach to saturation.}

Lamont's Law [15], for approach to saturation may be set out as:

$$
\frac{J}{J_{S a t}}=\frac{H}{H+C}
$$

where $\mathrm{C}$ is the so called magnetic hardness

Comparing this with actual measurements gives the results shown in Fig. 6, and the dependence of $1 / \chi$ is shown in Fig. 7.

Good constancy of the derived values of $\mathrm{C}$ was obtained so that it was calculated that $\mathrm{J}_{\text {sat }}$ could be obtained with a standard error of $0.03 \%$. The absolute value of $\mathrm{J}$ is of course limited by the precision of the assumed ultimate value of $\mathrm{J}_{\text {sat }}$.

Separate values of $\mathrm{C}$ were derived for Grain-Oriented (G.O.) and Non-Oriented (N.O.) steel. For the G.O., the value of C is 799, and for the N.O. sheets C is 248 .

There did not seem to be a need to devise or use constants

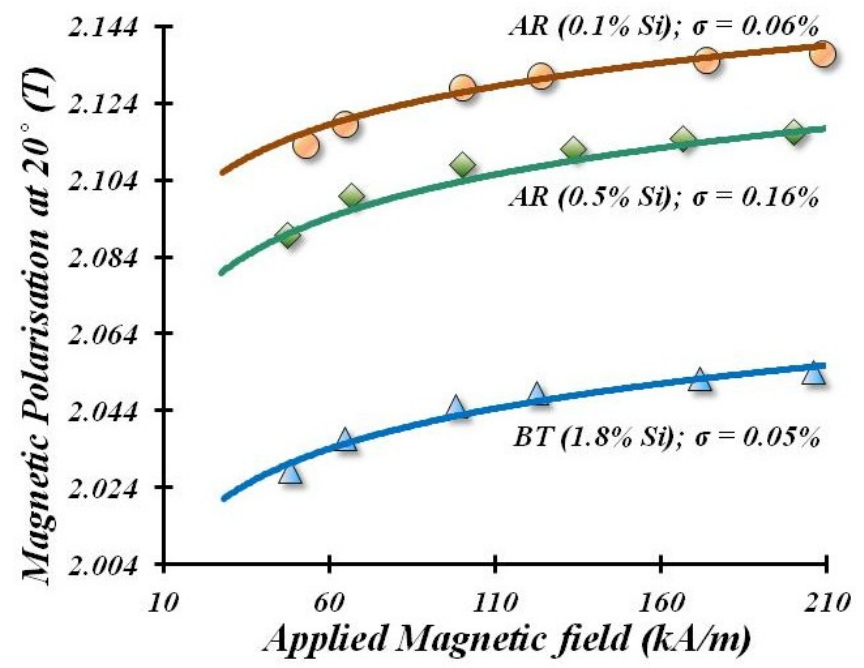

Fig. 6. Magnetic Polarisation against applied magnetic field for batches of three grades of non-oriented steels, measured at $20^{\circ} \mathrm{C}$. $A R$ and $B T$ are alphabetical codes used to describe the sample batches $A$ and $B$, and letters $R$ and $T$ in the code refer to rolling direction and transverse to rolling direction, respectively.

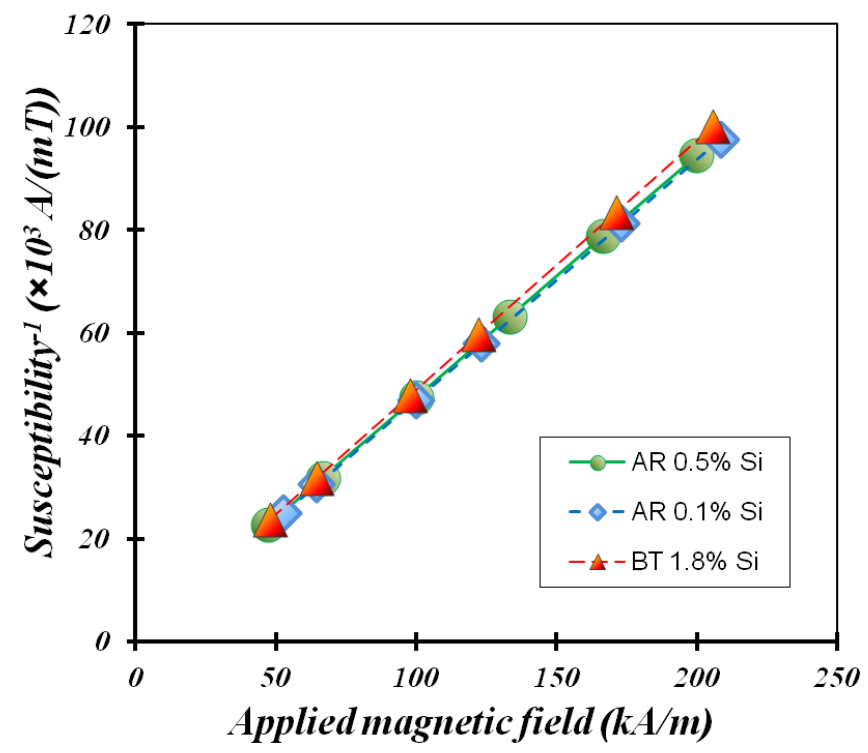

Fig. 7. Susceptibility against applied magnetic field for batches of the same three grades of non-oriented steels, measured at $20^{\circ} \mathrm{C} . A R$ and $B T$ are alphabetical codes used to describe the sample batches $A$ and $B$, and letters $R$ and $T$ in the code refer to rolling direction and transverse to rolling direction, respectively.

more specifically aimed at different grades of Non-Oriented steel; 248 was observed to fit them all adequately.

\section{ALLOY DEPENDENCE OF $\mathbf{J}_{\mathrm{sat}}$}

Combining the data from some 440 samples a relationship of the form $\mathrm{J}_{\mathrm{sat}}=2.132-0.041(\% \mathrm{Si}+0.73 \% \mathrm{Al})$ emerged. The effect of other elements was observed to be minimal in all cases. Only the effects of silicon and aluminium contents are usually considered. But in the widely used non-oriented and grain-oriented silicon-iron steel there are other elements like manganese and cobalt. A full study was reported in [16].

\section{COMPARISON OF THREE METHODS FOR THICKNESS DETERMINATION}

A very large amount of data was analysed to give an overall assessment of the comparability of methods. The saturation magnetic polarisation and $\gamma$-ray methods agree very well; and a constitutional difference of $-0.1 \%$ could be offset as required. These methods are of course in contrast with, and for the replacement of the conventional density method [1], which would be impractical to use on production line, since it would require the weight of the steel sheet being produced at high speed.

It is clear that the limiting circumstances hindering the precise evaluation of saturation magnetic polarisation with respect to other methods lies in the intrinsic uncertainties of those methods. In other words, $\mathrm{J}_{\text {sat }}$ based assessment is at least as good as, and probably better than, existing procedures.

As greater and greater inter-comparative resolution is sought all three methods disappear into the noise emanating from alloy uncertainty and actual spatial variation of thickness. 
The benefits of using saturation magnetic polarisation appear to be; (i) Avoidance of high voltages and isotope sources, (ii) Inherently high accuracy, (iii) Direct access to measurement of cross section, (iv) Applicability to ferromagnetic materials other than Electrical Steels, (v) When combined with a length integral for strip it gives direct access to tonnage passing through production line, (vi) Low first cost and maintenance, (vii) No need for whole system of precision off line standards.

Disadvantages could be; Physical size (could be $0.5 \mathrm{~m}$ long on a strip line), Inability to produce across-strip thickness profiles (seldom done anyway).

\section{IMPLEMENTATION OF PRACTICAL SETUP}

To excite an Epstein size strip some 800 VA is required, but this is at a very low power factor and only some 75 watts of active is generally needed. For a 1 meter wide strip around $25 \mathrm{kVA}$ may be needed which would be equivalent to about 2 $\mathrm{kW}$ of active power. Suitable power factor correction capacitors would enable the load on supplies to be confined to watt-delivering current. No sophisticated waveform or frequency control is needed, and as long as the frequency is noted by the controlling microprocessor, ordinary mains supplies will be quite sufficient for energizing the system. There would also be no need for any special voltage regulation requirements. It is also possible to take measurements of a few cycles only at intervals, to further reduction of the power consumption. Non strip-enwrapping versions may also be practicable.

\section{CONCLUSIONS}

Magnetic saturation has long been thought of as a means by which precision cross sectional area could be assessed. It has required an extensive study to lift the enquiry from a quick look at a few samples under ill-defined conditions to become a full examination which makes clear all the reasons for the behaviour observed.

It is clear that a fully satisfactory method of magnetic cross sectional area assessment is both possible and practicable along with hitherto unavailable precision, which is well above the minimum requirements for industrial exploitation.

\section{ACKNOWLEDGMENT}

The author wishes to thank Professor P. Beckley, Dr. H. Stanbury and Dr. D. Snell (previously with Orb and European Electrical Steel, Newport, UK) for their helpful discussions and preparation of large quantities of samples. The development of the initial system and measurements carried out were mostly the result of dedicated and hard work by Dr. J.Z. Cao during his postgraduate studies.

\section{REFERENCES}

[1] BS: Magnetic materials - Publication 6404: Part 21996 (also - BS EN 60404:13:2007 and IEC-60404-13:2007)

[2] Mthombeni, T., Pillay, P., "Lamination Core Losses in Motors with Nonsinusoidal Excitation with Particular Reference to PWM and SRM Excitation Waveforms," IEEE Trans. on Energy Conversion 20 (2005) 836-843.

[3] Kwon, S.O., "Loss Distribution of Three-Phase Induction Motor and BLDC Motor According to Core Materials and Operating," IEEE Trans. Magn. Vol. 45, No. 10 (2009) 4740-4743.

[4] Zou L., et al., "Impact of Saturation Depth Ratio of Iron-Core on the PMFCL," International Conference (ICEMS 2008) on Electrical Machines and Systems (2008) 4338-4343.

[5] Wang, X., Xie, D., "Analysis of Induction Motor Using Field-Circuit Coupled Time-Periodic Finite Element Method Taking Account of Hysteresis," IEEE Trans. Magn. Vol. 45, No. 3 (2009)1740-1743.

[6] Chen, J., Ma, W., et al, "Development Of Automatic Iron Loss Measurement System of Magnetic Material With PWM Excitation," 2011 IEEE International Conference on Materials for Renewable Energy \& Environment (ICMREE) (2011) 1013-1017.

[7] Kefalas, T.D., Kladas, A.G., "Analysis of Transformers Working under Heavily Saturated Conditions in Grid-Connected Renewable Energy Systems," IEEE Trans. Ind. Electronics Vol. 59, No. 5 (2012), 23422350 .

[8] Stumberger, G., "Prevention of Iron Core Saturation in Multi-Winding Transformers for DC-DC Converters," IEEE Trans. Magn. Vol. 46, No. 2 (2010) 582-585.

[9] Klopi, B., et al., "Analysis of an inverter-supplied multi-winding transformer with a full wave rectifier at the output," Journal of Magnetism and Magnetic Materials 320 (2008) 2929-2934.

[10] Jaiswal, V., et al., "Accurate Performance Prediction of Three-Phase Induction Motor by FEM Using Separate Saturation Curves for Teeth and Yoke," PEDES (Power Electronics, Drives and Energy Systems) '06 International Conference (2006)1-4.

[11] Kim, K., et al., "Characteristics of Ferromagnetic Materials Due to Uneven Magnetic Saturation," IEEE Trans. Magn. Vol. 47, No. 10 (2011) 3669-3672.

[12] Zhang, C., et al., "Magnetic characterization of surface-hardened steel," Journal of Magnetism and Magnetic Materials 321 (2009) 3878-3887.

[13] Hauser, H., et al., "An approach to modelling the dependence of magnetization on magnetic field in the high field regime," Journal of Magnetism and Magnetic Materials 300 (2006) 273-283.

[14] Beckley P., Master standards for radiation thickness gauging., Sheet Metal Industries, pp.598-601, 1974.

[15] Lamont J., Handbuch des magnetismus., Voss Leipzip, pp.1-468, (1867).

[16] Shirkoohi, G.H., "Dependence of Magnetisation near Saturation on Alloying Content in Ferromagnetic Steel," IEEE Trans. Magn. (2015) (in press)

[17] Shirkoohi, G.H., Cao, J.Z., "Thickness evaluation for steel sheets uses saturation magnetisation," Review of Progress in Quantitative Nondestructive Evaluation, Vol. 17 (1998) 1501-1508. 